\title{
Cefdinir Induced Red Stool: Harmless Antibiotic Side Effect Awareness for Parents
}

\author{
Trishna Sharma MBBS, MPH* and Riwaaj Lamsal MD ${ }^{1}$ \\ ${ }^{1}$ Email: riwaajlamsal@gmail.com \\ *Corresponding author: Trishna Sharma, MBBS, MPH, USA. Email: drtrishnasharma@gmail.com
}

\section{ARTICLE INFO}

Received: 幽 April 27, 2020

Published: 㠦 May 07, 2020

Citation: Trishna S, Riwaaj L. Cefdinir Induced Red Stool: Harmless Antibiotic Side Effect Awareness for Parents. Biomed J Sci \& Tech Res 27(3)-2020. BJSTR. MS.ID.004514.

Keywords: Cefdinir; Red Stools; Iron; Infant Formula
Abstract

Background: Cefdinir, an extended spectrum third generation cephalosporin is very commonly used in paediatric population. The American Academy of Paediatrics (AAP) recommends it as an alternative agent for initial treatment of acute otitis media in penicillin allergic patients. One of its harmless side effects is reddish coloured heme negative stools due to interaction with iron and iron containing products. This side effect is reversible upon drug discontinuation. However, dearth of literature and case reports on this common side effect has led to inattention to this issue and is not communicated to parents and caregivers. When encountered with bloody stool during the course of treatment with cefdinir, high concern from parents lead to unnecessary emergency room visit. This adds burden to already overworked health system escalating the practice of cost un-effective medicine.

Case Presentation: We present an 8-month old infant girl currently treated with Cefdinir and being formula fed, who presented to the Emergency Department and was further evaluated for red stools. On systemic examination, gastrointestinal findings were normal and hemoccult test of the stool returned negative for heme.

Conclusion: Cefdinir is commonly prescribed for infants who are also commonly on iron fortified diet. Paediatricians should expect the occurrence of red coloured stool once therapy with cefdinir is started and inform about the potential side effect that is not dangerous. This timely communication can save from unnecessary emergency room visits. Reporting this case adds to the scanty literature information we have on one of the common, and harmless side effects of cefdinir.

\section{Introduction}

An 8-month old female infant was brought to the ED with concern of one episode of blood in the stool. According to the mother, she noticed large amount of red coloured stool while changing the infant's diaper. The blood was bright red in colour, 3-4 tablespoon in amount and mixed with minimal mucus. There was a history of slight decrease in appetite but otherwise, the child was doing fine and passing normal amount of urine. The child was formula fed and on rice cereals. There was no concern of abdominal distension, vomiting, fever or irritability. The caregiver did give us a history that the infant had been seen at an urgent care facility 3 day ago for runny nose, cough and ear pulling. She was diagnosed with ear infection and was prescribed with Cefdinir which the child was currently on.

Past medical and surgical history was non-contributory, and immunizations were up to date. On general examination, the infant was slightly irritable but in no acute distress. The neck was supple without lymphadenopathy and both the right and left ear examination revealed normal tympanic membrane. On gastrointestinal examination abdomen was soft, non-tender and non-distended with normal bowel sounds. There was no organomegaly or palpable mass and rectal examination was also normal. Other systematic examination revealed normal findings 
and hemoccult test was done from the sample of stool brought by the mother which came out to be heme negative when tested twice (Figure 1).

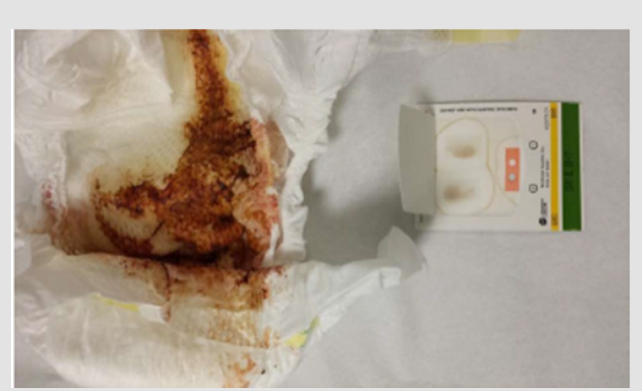

Figure 1: Red Stools as shown above, however Hemoccult negative.

\section{Final Diagnosis}

Cefdinir induced red stool.

\section{Discussion}

Cefdinir, a commonly used third generation cephalosporin has extended range of coverage for Gram negative and Grampositive bacteria [1]. The popularity of its use is mostly because of advantages like less frequent dosing, shorter therapy and its superiority to penicillin in case of resistance.1-2 It is also highly rated antibiotic according to provider preference [2]. However, one of Cefdinir's harmless yet alarming side effects of red coloured nonbloody stool is less reported in literature. Our purpose of reporting this case is to make readers aware that conveying a simple information about a benign drug side effect may be helpful in adherence to therapy.In 2000, a case report described 2 such cases in which Cefdinir use for infection in a child with otitis media and in a child with bronchopulmonary dysplasia led to the occurrence of red stools.Both these children were receiving iron containing elemental formula [3].

Another case report on the same issue was made in 2008. A 7-month old infant with a history of apnoea of prematurity and dietary anaemia presented to the ED with concern of 2 episodes of large red stools. Physical examination was otherwise unremarkable except for a small anal fissure. Extensive workup was done for the patient which was negative, and the patient was discharged to be managed on the line of constipation. There was recurrence of the bloody stool and at this time, on careful history taking, it was found that the child was being treated with Cefdinir for otitis media [4].

Lancaster et al reported a case in 2008 about a 9-month old child with acute otitis media who was switched to Cefdinir from the initial antibiotic for concern for allergic reaction. Subsequently, the child developed red stools prompting work up. Upon discontinuation of Cefdinir, his stools returned to normal within 48 hours. There was dietary history of being breast fed. Three weeks later, another episode of otitis media occurred and given the known history of allergic reaction to penicillin he was again started on Cefdinir. After 48 hours, red stools appeared again and were guaiac negative and this resolved upon drug discontinuation. Here, both the recurrences were due to concomitant administration of Cefdinir and iron containing infant formula. Since, the infant was also formula fed along with breast milk this crucial part of the history was missed [2].

Lowers et al in 2009 have given 4 cases who presented with visible red stools [5]. Initial history here included dietary history of formula food fortified with iron or food with edible colouring agents as ingredient. Stool guaiac test was positive only in one case, which later turned out to be intussusception. The second case, on further questioning of dietary history revealed an intake of $16 \mathrm{oz}$. of red cake frosting the day before presentation. The third case was Cefdinir induced harmless drug-diet interaction. The fourth case revealed ingestion of strawberry coloured drink by the infant. It was presumed that the sugar load from the drink may have caused an osmotic diuresis, fastening stool transit time which rendered artificial coloring to remain unchanged by digestion.

This red colour is due to formation of a non-absorbable complex between Cefdinir or it's metabolites with iron present in the gastrointestinal tract [6]. The incidence of this occurrence seems to be variable. Graves et al. have reported in their study an incidence of $1 \%$ whereas Nelson JS report it to be $0.2-0.3 \%[3,4]$. The onset of red stools in our case report was approximately 48 hours which is similar to what other studies have also reported $[2,3]$. Such temporary reddish discoloration can be reversed upon discontinuation of the drug and is harmless. Physicians should also be aware of drugs that can lead to Orange-red stools (Phenothiazine, Rifampin) and Pink-red stools (Barium, Hydroxycobalamin, Rifabutin, Senna, Tetracycline) [7]. Awareness about these drug interactions is important as it will improve the drug compliance and also avoid unnecessary workup [3].

In all above cases including our case report, the Naranjo adverse drug reaction probability scale was used to assess that Cefdinir being the cause behind red coloured heme negative stools was highly probable. The packages insert in Cefdinir mentions important information about iron supplements and iron containing products [4].

\section{For Iron Supplements}

It is suggested that keeping an interval of 2 hours before or after taking iron supplement or iron containing multivitamins along with Cefdinir. This concomitant intake of iron supplement (containing $60 \mathrm{mg}$ of elemental iron) and multivitamins (containing $10 \mathrm{mg}$ of 
elemental iron) with Cefdinir decreases the extent of absorption of Cefdinir by $80 \%$ and $31 \%$ respectively.

\section{For Iron Containing Products}

Iron containing products like iron fortified infant formula (2.2 mg elemental iron/6oz.) has nosignificant effect on Cefdinir pharmacokinetics and can be administered with Cefdinir.

\section{Conclusion}

Infants and children are vulnerable to risk for upper respiratory tract infection and hence use of prescription antibiotic appears to be common among them. Physicians prescribing Cefdinir should make a note of children taking iron supplements and foods fortified with iron and pre-inform parents about possibility of passage of harmless red coloured stools. Therefore, while prescribing Cefdinir, a complete dietary and nutritional history becomes a crucial part of evaluation. Simple knowledge and awareness about drug- diet benign interaction between Cefdinir and iron containing products will save a patient from costly investigations for suspected gastrointestinal bleeding. This important awareness will also increase drug compliance as it is equally important to treat the underlying infection and solve issues of antibiotic resistances. As long as prescription medicines are used keeping in mind that benefit outweighs risk, and, in this condition, red coloured stools are just a side effect and not an adverse drug reaction, the therapy should definitely be encouraged to continue until required.

\section{Ethics Approval and Consent to Participate}

$$
\mathrm{N} / \mathrm{A}
$$

\section{Consent for Publication}

$$
\text { Yes }
$$

ISSN: 2574-1241

DOI: $10.26717 /$ BJSTR.2020.27.004514

Trishna Sharma. Biomed J Sci \& Tech Res

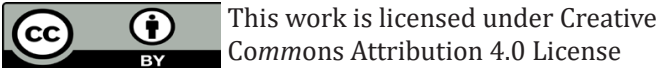

Submission Link: https://biomedres.us/submit-manuscript.php

\section{Availability of Data and Material}

N/A

\section{Declaration of Competing Interest}

The authors of this manuscript have no significant professional, financial and personal interest that might have influenced the performance or presentation of the work described in this manuscript.

\section{Funding}

N/A

\section{Authors Contribution}

Literature review, writing, editing and preparing manuscript

\section{Acknowledgement}

None

\section{References}

1. Guay D (2000) Pharmacodynamics and pharmacokinetics of Cefdinir, An oral extended spectrum cephalosporin. Pediatr Infect Dis J 19: S141-146.

2. Lancaster J, Sylvia LM, Schainker E (2008) Nonbloody, red stools from coadministration ofCefdinir and iron-supplemented infant formulas. Pharmacotherapy 28(5): 678-681.

3. Nelson JS (2000) Red stools and omnicef. J Pediatr 136(6): 853-854.

4. (2008) Omnicef package insert. Chicago, IL: Abbott Laboratories.

5. Lowers J, Jaffe A, Zenel JA, Cabana MD, Donahue C, et al. (2009) Visual diagnosis: four infants who have red, "bloody" stools. Pediatr Rev 30(4): 146-149.

6. Graves R, Weaver SP (2008) Cefdinir-associated "bloody stools" in an infant. J Am Board FamMed 21(3): 246-248.

7. Roath MC, Di Palma JA (2013) Correspondence: cefdinir and red stool. Gastroenterol Hepatol (NY) 9(6): 338.

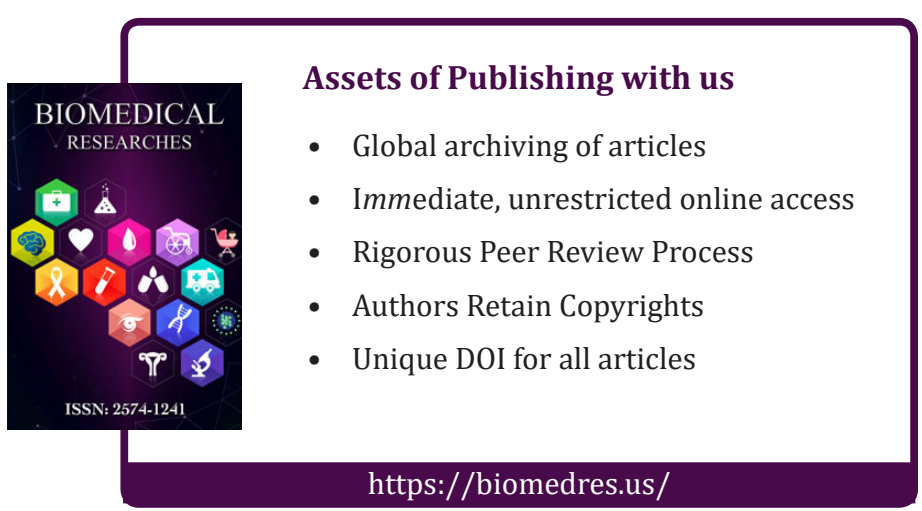

